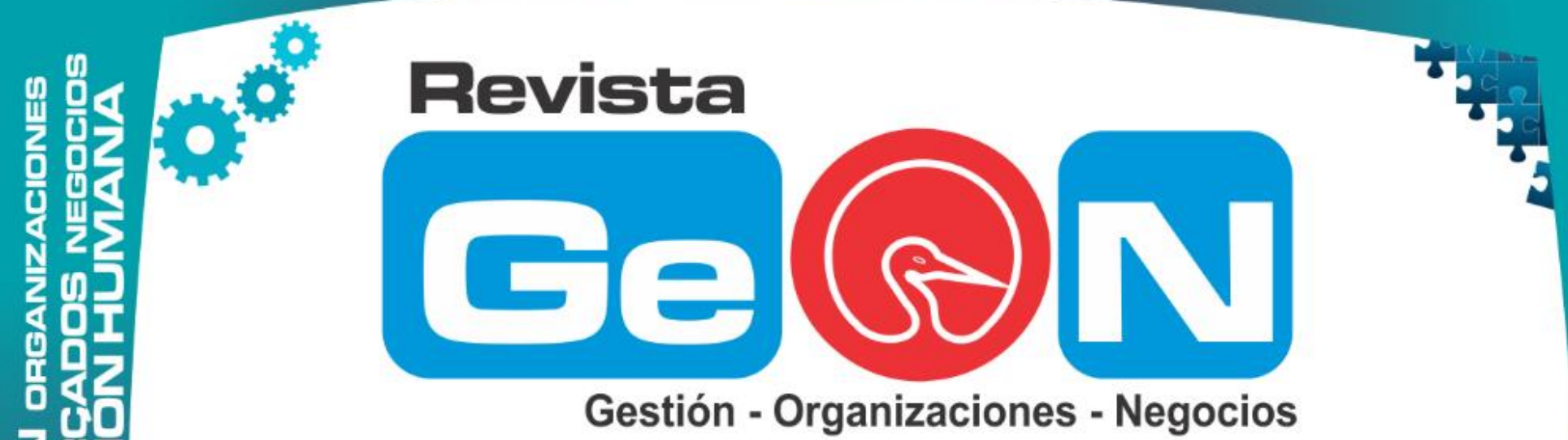

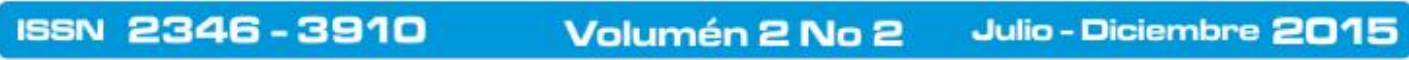

Revista Electrónica de la Facultad de Ciencias Económicas de la Universidad de los Llanos

1. 3 II

440

VILLAVICENCIO - COLOMBIA

$>2$

64

2

2 平品

70

ব)

展

o

ii III

0 U

ใด

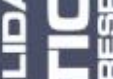

居的

임

Iiin in

照

只

造

iil 2

if in

4 吕

2

400

付 iI
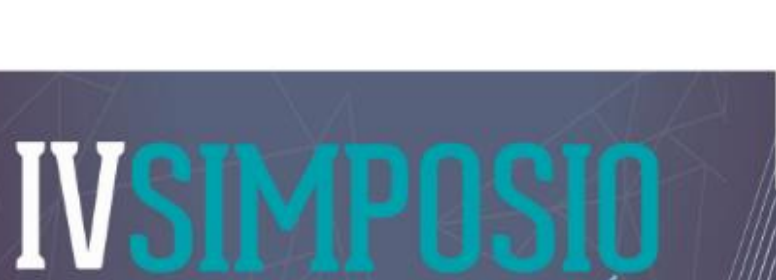

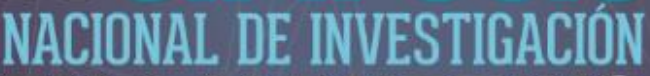

EN MARKETING
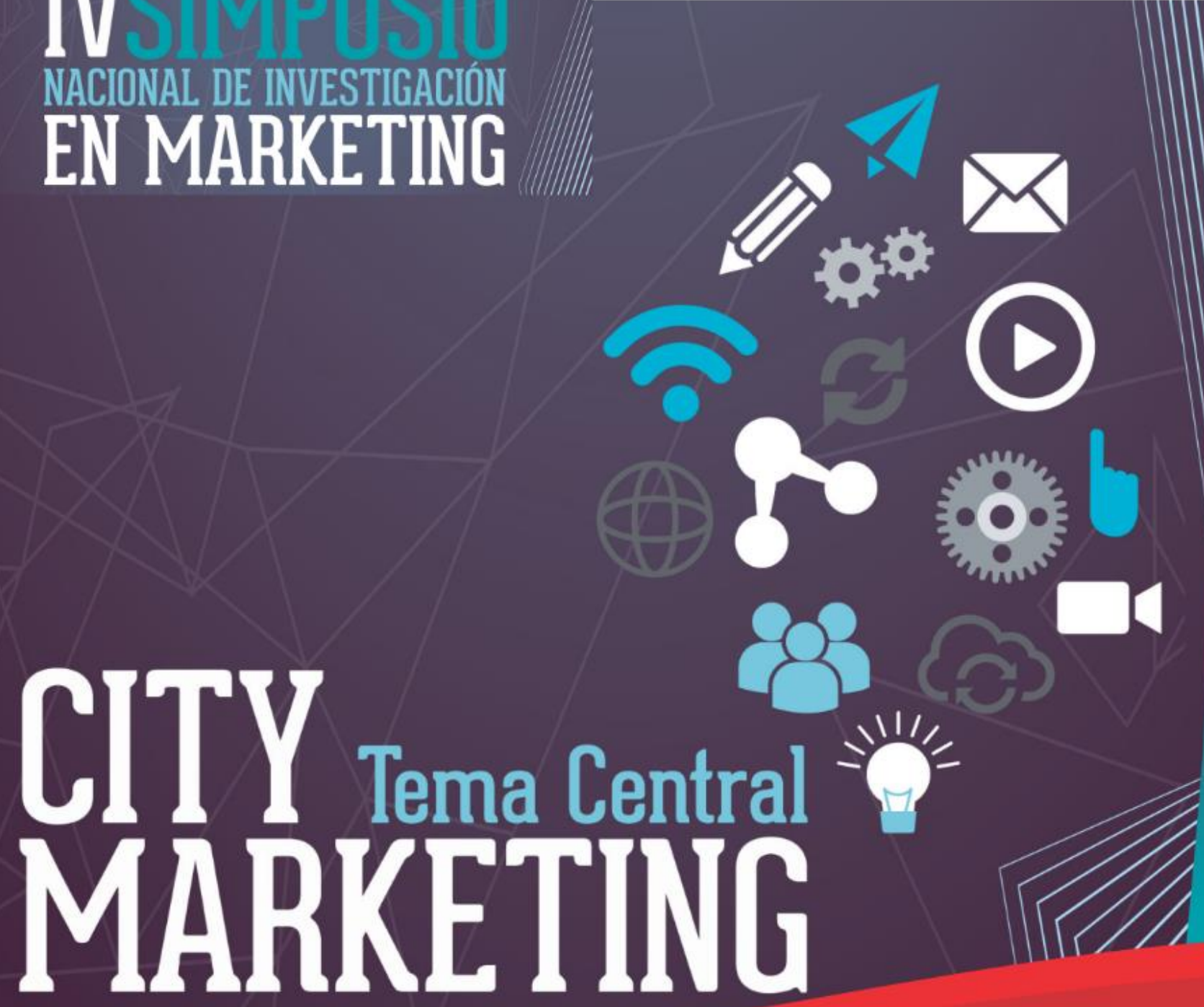

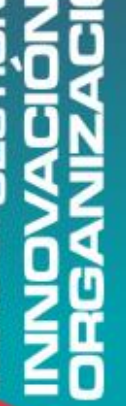
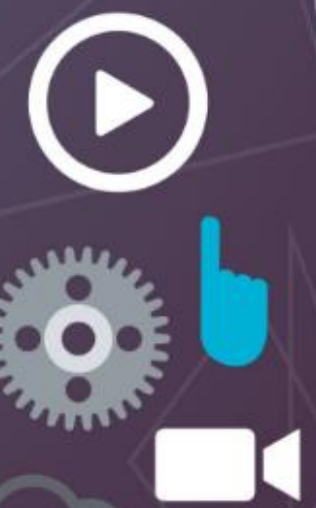


\section{Lineamientos para el desarrollo sostenible del sector agropecuario en Colombia - Jairo Vásquez}

Jairo Alberto Vásquez Bernal

(C) Magister Dirección de marketing Especialista Gestión Social y Ambiental, jvasquez@uniagustiniana.edu.co

\section{Resumen}

En el siglo XXI debido al desarrollo tecnológico se han presentado transformaciones sociales, demográfica y económicas, y en un intento por asegurar que los cambios que afectan la humanidad sean para mejorar, sin deteriorar el ambiente, se fomenta el desarrollo sostenible, el cual se orienta en la preservación, manejo, y utilización adecuada de los recursos naturales, los ecosistemas y la habitabilidad.

El desarrollo sostenible es un concepto relativamente nuevo, por lo cual se hace necesario que se indague por lo que realmente significa y por la manera de aplicarse, por lo cual, en primera instancia se hace un exploración bibliográfica en el que se evidencien los diferentes puntos de vista y se establecen los elementos que posibilitan su comprensión y apropiación del concepto; lo anterior, permite la determinación de los lineamientos básico para el desarrollo sostenible orientado a mostrar la justificación y propósitos para alcanzar un entendimiento común respecto a este tema, estructurado directrices básicas para que las empresas del sector agropecuario trabajen con prácticas ambientalmente responsables, protegiendo la biodiversidad y consiguiendo sostenibilidad.

\section{Palabras clave}

Desarrollo sostenible; biodiversidad; recursos y sector agropecuario.

\section{Abstract}

In the XXI century due to technological development have presented social, demographic and economic changes, and in an attempt to ensure that changes affecting humanity are to improve, without damaging the environment, promoting sustainable development, which aims in the preservation, management, and appropriate use of natural resources, ecosystems and livability.

Sustainable development is a relatively new concept, so it is necessary to inquire by what it really means and how to apply, therefore, primarily an exploration literature in which the various points made evidencing view and elements that enable comprehension and appropriation of the concept are established; above, allows the determination of the basic guidelines for sustainable development aimed at showing justification and purpose to achieve a common understanding on this issue, structured basic guidelines for businesses of the agricultural sector to work with environmentally responsible practices, protecting biodiversity and achieving sustainability

\section{Keywords}

Sustainable development; biodiversity; resources and agricultural sectors.

\section{Introducción}

A partir de la segunda mitad del siglo XX, se han utilizado los recursos naturales renovables $\mathrm{y}$ no renovables sin control, generando impactos ambientales, especialmente en el sector agropecuario y la biodiversidad, esto se debe al desarrollo tecnológico en informática, telecomunicaciones, robótico, electrónica y genética que han generado transformaciones sociales, culturales, demográficas, tecnológicas y económicas. Esto con el fin de mejorar la calidad de vida y la satisfacción de las necesidades de la comunidad, a partir de entonces se genera el desarrollo sostenible, el cual establece que se puedan satisfacer las necesidades básicas de supervivencia y de bienestar de las generaciones 
presente $\sin$ afectar el de las generaciones futuras.

Es importante indicar que el desarrollo sostenible es un concepto relativamente nuevo, y se define como aquél desarrollo que es capaz de satisfacer las necesidades actuales sin comprometer los recursos y posibilidades de las futuras generaciones. Intuitivamente una actividad sostenible es aquélla que se puede mantener (Burton, 1987). Principios que se proponen para el sector agropecuario.

Este artículo tiene como objetivo proporcionar lineamientos que favorezcan el desarrollo sostenible de las cadenas del sector agropecuario en Colombia. El trabajo se plantea bajo un marco amplio que tienen en cuenta los referentes de la literatura, diseño y elaboración de matrices, procedimientos y un diagnóstico de la problemática agropecuaria nacional. Los resultados muestran la evolución conceptual, los elementos, componentes y características del desarrollo sostenible y los fundamentos, del desarrollo sostenible, igualmente los lineamientos para orientarlos al sector agropecuario.

La República de Colombia se ubica en la esquina noroccidental de Suramérica con una extensión territorial de 2.070.408 km2 (2.022.124) incluidas las áreas terrestres $(1.141 .748 \mathrm{~km} 2)$ y marinas $(880.376 \mathrm{~km} 2)$. El país ocupa el cuarto lugar en extensión entre los países de América del Sur, el séptimo en América y el 25 en el mundo (IGAC, 2003) En esta área se encuentra el $10 \%$ de la biodiversidad mundial, haciendo de Colombia un país «megadiverso» (MMA, DNP, IAvH, 1998).

Colombia es un país megadiverso, sin embargo, esta inmensa ventaja comparativa prácticamente no ha repercutido notoriamente en su desarrollo económico, entre otras cosas porque se desconoce cómo conformar y desarrollar los sistemas de comercialización para productos de la biodiversidad para beneficiar al sector agropecuario.
Pero como lo expresa Nadal (2001), hoy la crisis del sector agropecuario está marcada por tres rasgos distintivos. Primero, el entorno macroeconómico es más desfavorable y eso se traduce en múltiples efectos negativos Segundo, se presenta un retiro casi completo del apoyo estatal en todos los renglones. Tercero, la crisis del sector agropecuario estalla precisamente en el marco de una apertura acelerada, indiscriminada y que se aplica de una manera irresponsable, rompiendo los compromisos que el gobierno había adquirido con los productores nacionales; situación vigente, pero existen posibilidades de mejora.

Otros problemas son los relacionados con los tratados de libre comercio, ya que en los países industrializados el sector agropecuario es subsidiado, y existen políticas definidas de seguridad alimentaria, y se ratifica con lo expresa Cortes (2004). La apertura económica con su acelerado crecimiento de las importaciones tampoco ha logrado garantizar la buena alimentación.

Los problemas del sector agropecuario se deben entre otras razones: al hecho de cultivar regularmente lo mismo; maíz, papa y hortalizas, lo cual, hace que cada vez se utilicen más fungicidas, herbicidas insecticidas, abonos $\mathrm{y}$ fertilizantes, sin mejorar la productividad, lo cual influye en los costos disminuyendo la rentabilidad de los productos para los cultivadores y no para los intermediarios.

También, por que los pequeños productores siembran por tradición y por el conocimiento de una manera de cultivo, pero no tienen en cuenta proceso para el desarrollo sostenible del sector agropecuario, ya se utilizan los recursos naturales indiscriminadamente y se desconocen sus potenciales.

Pero, el problema que más afecta actualmente a los campesinos, es desconocimiento de alternativas de cultivos $\mathrm{y}$ comercialización para los productos, ya que la mayoría de veces se cultiva lo mismo y se venden en las centrales de abastos, donde tienen 
poco poder de negociación, ya que la condiciones, cantidades y forma de pago las establecen los intermediarios, además se desconoce cuáles son las necesidades de la demanda.

Con el fin de comprender la situación y presentar los lineamientos, se realizará una investigación de tipo aplicada, ya que corresponde a las ciencias económicas y administrativas en la cual se desarrollará el proyecto, con enfoque de investigación cualitativa a través de procesos de investigación descriptiva y documental.

La investigación es descriptiva, según Tamayo y Tamayo (2002), ya que se aplica el análisis de datos con los cuales se presentan los fenómenos o hechos de la realidad que dada su similitud, es necesario describir sistemáticamente a fin de evitar un posible error en su manejo; es decir, se presentan los resultados en tablas, graficas que describen los resultados se analizan e interpretan relacionadas con las actividades turísticas, tipo, ecoturismo, municipios visitados, frecuencia de visitas, inversión e interés por participar en el corredor turístico y en la empresa administradora.

\section{Investigación Documental}

La información recolectada a través de la investigación documental se realizó por medio de consultas de libros, registros, constituciones y estudios de turismo. En estos documentos se encontró una variedad de información sobre desarrollo sostenible, la cual permitió sentar las bases del enfoque del proyecto y permitió desarrollar a cabalidad el objetivo de la investigación. Los términos encontrados permitieron aclarar y unificar las definiciones que eran necesarias para evitar fusiones el momento de determinar los lineamientos.

\section{Desarrollo}

La Economía mundial se caracteriza por los avances tecnológicos, principalmente en informática, electrónica, robótica, genética y nanotecnología, también por la globalización, la integración y la apertura económica, lo que hace que los procesos comerciales sean dinámicos y estén en permanente cambio, lo mismo que los hábitos de compra y uso de bienes y servicios. Esto se ratifica con lo expresado por Schnarch (2009),"vivimos en una época de cambio acelerado. Tradicionalmente este cambio era evolutivo, seguro, episódico y programable, pero actualmente es acelerado, incierto, continuo e impredecible".

También, los empresarios como lo expresa Nail (2013), notaron que en los últimos 10 años se han producido una gran cantidad de cambios, ya sea por la transformación del mercado de las necesidades de los clientes o de las condiciones económicas, no hay dudas que la tecnología ha sido fundamental para llevar adelante todos estos cambios; ha modificado nuestras vidas personales $y$ se ha infiltrado en nuestras actividades diarias, acelerando nuestro ritmo de vida y ofreciéndonos más posibilidades que nunca, lo cual hace que las necesidades y deseos se incrementen.

Es importante destacar que el desarrollo económico actual se fundamenta en la generación de riqueza, en estándares de calidad de vida, pero los resultados ambientales de este tipo de desarrollo son los siguientes:

Las emisiones de CO2 están aumentando a nivel global, pasando de 26,4 toneladas métricas a 28,26, entre 1990 y 2010, lo que afecta la temperatura y tiene relación directa con el cambio climático, lo que impacta especialmente la producción agropecuaria. El cual también se afecta por el uso de fertilizantes y comparando las diferentes regiones donde se encuentran la mayoria de países, Colombia es el que mas utiliza, pasando en 10 años de 304 kilogramos por hectarea a 744, lo que disminuye la productividad.

Además, el uso de agua, en las cantidades actuales, afecta el entorno, ya que cada vez se necesita mas agua, pero ademas se contaminan 
las quebradas y rios por las aguas residuales, ya que no son tratadas adecuadamente.

Lo anterior influye en Producto Interno Bruto PIB de la agricultura, esepcialmente en Colombia ya que paso de 8,9 a 6,1 , es decir un perdida de 2,8 puntos del PIB, lo que muestra que el sector no es sostenible.

Esto también, se ve reflejado, en la cantidad de personas que se encuentran en el sector rural, en colombia del 2000 al 2013, paso de 27,96\% a 24,117, situacion dada ademas por el conflicto interno, por lo poca productividad $\mathrm{y}$ sostenibilidad del sector. Mientras que el indice de produccción de alimentos aumenta en todos lo paises incluyendo Colombia, los resultados no se evidencian en aumento de lass exportaciones de productos, ya que la balanza comercial es negativa, ya que se importa más de lo que se exporta especialmente con el principales socios comerciales Estados Unidos, y con la Unión Europea.

Es importante indicar que la agricultura es uno de los principales motores del desarrollo económico. Pero las Políticas macroeconómicas son las que directamente afectan a través de la influencia en los precios relativos en el sector. (Norton, 2014). Transformando el sector agropecuario se pueden ofrecer productos con valor agregado para muchos sectores, como el sector farmacéutico, fitomedicinas, medicinas tradicionales, agroquímicos, cosméticos, fragancias, sabores, nuevos materiales, pero además desarrollar procesos de investigación, para generar conocimientos que permitan ser una potencia mundial.

Esto se logra con procesos de desarrollo sostenible, iniciando con el uso adecuado al suelo, partiendo del uso actual, el cual no corresponde a las características del suelo, especialmente para el subsector pecuario ya que en el 2013 se utilizaron 30.362.649, y disponibles son 25.000.000 hectáreas, es decir 5.362649 hectáreas mal utilizadas, con un aumento 361.717 ha respecto al 2012, reduciendo el área agrícola y forestal, generando problemas de desarrollo sostenible.

Lo fundamental es preservar los recursos naturales y como lo expresa Fuentes \& casilla (1991) las consecuencias que todo esto tendrá sobre la humanidad futura nos son desconocidas, pero resulta claro que el modelo de crecimiento demográfico y socio-económico que se ha estado siguiendo no puede mantenerse a largo plazo, por lo cual, es necesario modificar el modelo, a una alternativa es el desarrollo sostenible ( $p$ 172).

Pero como lo expresa Bastian et al. (2007) "El desarrollo sostenible es un concepto controvertido, con las teorías conformadas por personas de diferentes visiones del mundo y de las organizaciones, que a su vez influyen en cómo se formulan las cuestiones y las acciones propuestas. Por lo general se presenta como la intersección entre el medio ambiente, la sociedad y la economía, que se concibe como algo separado aunque las entidades conectadas" ( $p$ 172).

El desarrollo sostenible no es preservar por preservar, implica tener en cuenta la interacción entre los ecosistema social, ambiental y económicos. "En términos generales, el concepto de desarrollo sostenible es un intento de combinar las crecientes preocupaciones sobre una serie de cuestiones ambientales con las cuestiones socioeconómicas" (Hopwood, et al. 2005).

Por lo cual, la consigna del desarrollo sostenible como lo expresa Huntley et al. (1991) La utilización de los sistemas ecológicos (la biosfera) se hace de tal de modo que permita satisfacer las necesidades actuales sin comprometer las necesidades u opciones de las generaciones futuras (p 228) por lo tanto a "largo plazo, la única opción que tiene sentido es procurar alcanzar la sostenibilidad del sistema socio ecológico completo. Las razones que justifican tener en cuenta el sistema como un todo es la existencia de importantes 
vinculaciones entre sociedad y naturaleza" (Gallopín. 2003. P15).

Es importante indicar que la ubicación geográfica de Colombia es una de las mejores del planeta, ya que se puede transportar productos, materias primas e insumos por el pacifico y el atlántico, del norte al sur, del oriente al occidente; cuenta con un área de 1.141.748 kilómetros cuadrados de superficie en la que habitan 48.321 .405 de habitantes (proyectado a 2013), por actividades antropológicas se disminuyó el área selvática en 22.220 kilómetros cuadrados en 22 años, es decir, en promedio por año se deforestan 1010 kilómetros cuadrados, para beneficio de unas comunidades y empresas, que promulgan el desarrollo económico actual, situación similar se presenta con los otros recursos naturales renovables y no renovables, pero esa riqueza no tiene una distribución equitativa y no se refleja en crecimiento ya que producto interno bruto que en promedio es de 4,8 .

Desde esta perspectiva son muchos los desafíos para al renglón agropecuario, entre los que se incluyen la vinculación de los pequeños productores a las cadenas de valor, poniendo los regímenes de tenencia de la tierra y políticas de gestión del agua sobre una base sólida, por lo que la agenda de investigación y extensión agrícola responde a las prioridades de los

Figura 1. Fundamentos del desarrollo sostenible. agricultores, la promoción de un mayor papel para el sector privado, fomentar el desarrollo sostenible de las finanzas rurales, y dar más oportunidades a las mujeres agricultoras (Norton, 2014).

Es importante ratificar que Colombia es uno de los países con mayor biodiversidad del planeta en el mundo con tan solo el $0,77 \%$ de superficie terrestre, cuenta con el $10 \%$ de las especies, y desde la celebración de la Conferencia de Río en 1992, se reconocen los derechos soberanos sobre la biodiversidad (Vallejo et al 2009), y en el país. Y que la alternativa de crecimiento es el desarrollo sostenible, a través de la utilización adecuada de los recursos y la distribución equitativa de la riqueza.

El desarrollo sostenible cuenta con varias ópticas, pero lo adecuado es la integración e interrelación entre los elementos del sistema lo social en donde interactúa el talento humano y el capital social, en lo económico con la generación de riqueza, prosperidad y bienestar y lo ambiental con los recursos naturales renovables y no renovables, fundamentado en la distribución equitativa de la riqueza, viabilidad en la preservación de los recursos y que se haga

factible el uso del medio ambiente sin que se destruya las diferentes formas de vida y que además las generaciones futuras, los puedan disfrutar, lo cual se deduce de la siguiente figura.

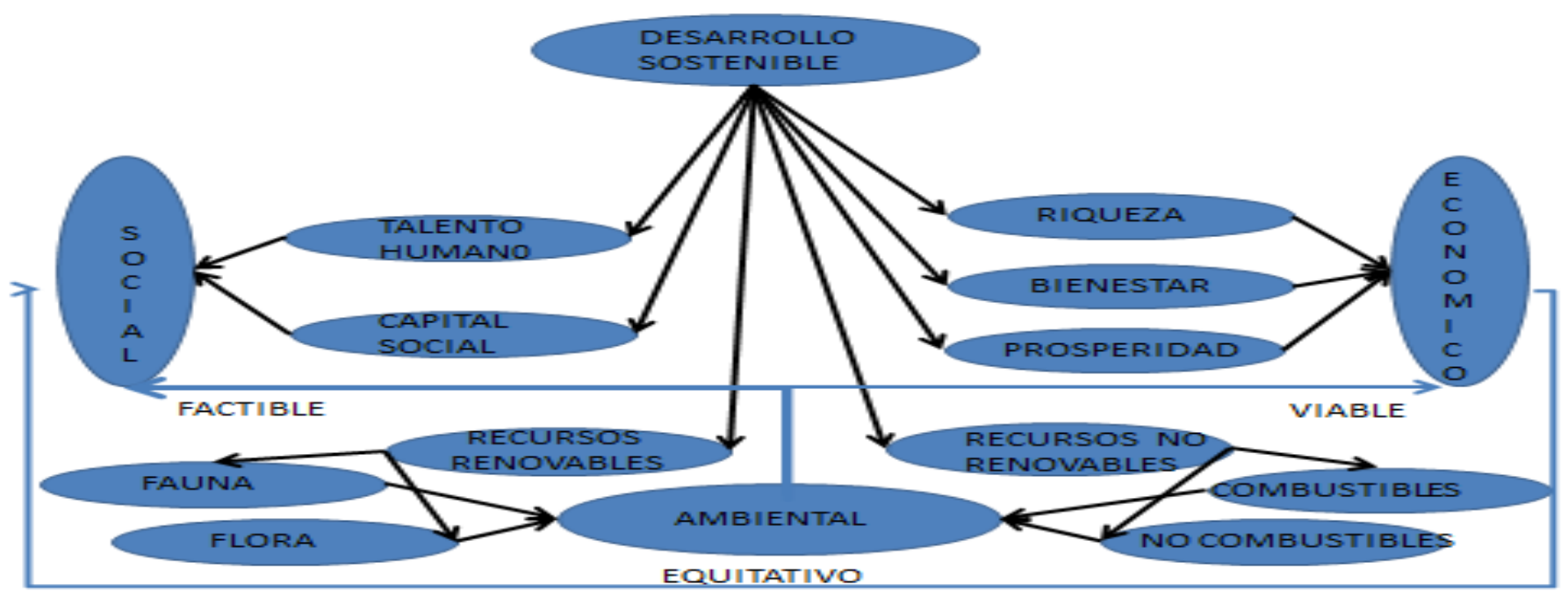


Lo anterior permite establecer los siguientes lineamientos para el desarrollo sostenible del sector agropecuario en Colombia.

Diversificación, es la estrategia de producto mercado más adecuada, ya que la riqueza bilógica del país permite ofrecer al mundo materias primas y productos con valor agregado en fitomedicinas, medicinas tradicionales, agroquímicos, alimentos nutricionales, cosméticos, fragancias, sabores, nuevos materiales, utilizando de manera adecuada la biodiversidad del país.

Multicultivos: cultivar simultáneamente varios productos, sin destruir los ecosistemas existentes, permitiendo la interacción de las plantas y bacterias para la fijación de nutrientes, trasformado los procesos de cultivo actual, arados parciales.

Biorremediación: utilización de plantas, insectos y microorganismos que controlan otros que afectan la productividad.

Economía social: el desarrollo económico se transforme fundamentado el bienestar del ser de la comunidad y del individuo.

Economía ambiental, los métodos de valoración de los precio y las estrategias se fundamenten el valor ambiental, no solo en el valor de las materias primas, el talento humano directo e indirecto y los costos fijos, sino incluyendo el valor de preservar los recursos.

Planificación de la producción y la comercialización: los estamentos públicos ministerios y el gobierno en general establezcan parámetros para la determinación de los cultivos, sus cantidades y tiempos a nivel nacional, con base en la demanda del mercado.

Subsidio de la formalización: es estado financie como empresa los impuestos o tributos que están cargados al salario, específicamente salud, pensión, ARL.

Reestructuración de la producción pasar de la labranza convencional con tractores y utilizar equipos como el retobo a podar y hacer en el terreno hoyos.

\section{Conclusiones}

La investigación es un aporte a los productores, comercializadores, compradores y consumidores, quienes dispondrán de herramientas para tomar decisiones de comercialización e incursionar con menor incertidumbre en los mercados potenciales que promuevan la preservación y manejo adecuado de los recursos.

De las definiciones de desarrollo sostenible establece que se integran las relaciones entre la sociedad, el medio ambiente y la economía, de tal manera que sea viable y sostenible la vida y que la distribución de la riqueza sea equitativa, preservando y utilizando adecuadamente los recursos para que las generaciones futuras también los puedan disfrutar.

Los lineamientos básicos del desarrollo sostenible se relacionan con la diversificación de los cultivos, especialmente orientados a la biodiversidad, los multicultivos, la biorremediación, la planeación de la producción y comercialización agropecuaria nacional y la estructuración de bases de economía social y ambiental.

\section{Referencias}

Bastian, O., Corti, C., Lebboroni, M. (2007). "Determining environmental minimum requirements for functions provided by agroecosystems": Agronomy for Sustainable Development, 27(4), 279-291.

Burton, I. (1987). "Report on Reports: Our Common Future: The World Commission on Environment and Development. Environment: Science and Policy for Sustainable Development", 29(5), 25-29.

Cortes, E. (2004) "Sector agropecuario y desarrollo rural: una mirada integral". Universidad Nacional de Colombia: la universidad. 274 p. ISBN 9587013530, 9789587013535 
Fuentes Eduardo R. \& Castilla Juan C. (1991) "Cambio Global, Desarrollo Sustentable y Conservación de la Biodiversidad: ¿Qué podemos hacer?" en: Revista Chilena de Historia Natural, 64:171-174,1991.

Gallopín, Gilberto. 2003. "Sostenibilidad y desarrollo sostenible: un enfoque sistémico" en CEPAL: DIVISION DE DESARROLLO SOSTENIBLE Y ASENTAMIENTO HUMANOS. Publicación de la naciones unidas: serie Medio Ambiente y Desarrollo 64: 9-17. 2003

Hopwood, Bill; Mellor, Mary; O'Brien, Geoff. (2005) "Sustainable development: mapping different approaches" [en línea], disponible en: Sustainable development. Volume 13, Issue 1, pages 38-52, February 2005

Huntley B.J.; Ezcurra E.; Fuentes E.R.; Fujii; K. Grubb. P.J; Haber; W.. Harger J.R.; Holland M.;. Levin S.A; Lubchenco J.; Mooney H.A.; Noble L.; Neronov; V. Pulliam R.H.; Ramakrishnan P.S.; Risser P.G.; Sala O.; Sarukhan J. Y. Sombroek W.G (1991) "Una Biosfera Sustentable: el imperativo global”, en : revista Chilena de Historia Natural 64: 227-235, 1991

IGAC - Instituto Geográfico Agustín 2003 "Atlas de Colombia. Bogotá D.C., Colombia, $224 \mathrm{p}$.
MINISTERIO DEL MEDIO AMBIENTE, Departamento Nacional de Planeación, Instituto Humboldt. 1998. "Política Nacional de Biodiversidad". Bogotá.

Nadal Alejandro (2001). "Programa Sobre Ciencia, Tecnología y Desarrollo. Documento De Trabajo NO. 1-05. Lineamientos de una estrategia alternativa de desarrollo para el sector agrícola". México: 2001 PROCIENTEC, El Colegio de México

Norton, R. (2014). "Policy Frameworks for International Agricultural and Rural Development. Texas (Estados Unidos)". Encyclopedia of Agriculture and Food Systems. Texas A\&M University, College Station, TX, 1 (2014) 489-503

Schnarch Kirberg, Alejandro. (2009). "Desarrollo de nuevos productos y empresas. Creatividad, innovación y marketing. Cómo generar, desarrollar e introducir con éxito". ( $5^{\mathrm{a}}$ ed). Bogotá: McGraw-Hill, 2009. 409 p.

Tamayo y Tamayo, Mario. 2002 "El proceso de la Investigación Científica”. 4 ed. México: Editorial Limusa Noriega Editores, 2002. p.42

Vallejo, f. Nemogá, r. Rojas,d. (2009) “Guía práctica para el acceso a los recursos biológicos, los recursos genéticos y/o sus productos derivados, y el componente intangible". Bogotá Colombia: Grupo PLEBIO - U. Nacional. 